\title{
Correction: Efficacy of toric intraocular lens implantation with high corneal astigmatism within the United Kingdom's National Health Service
}

\author{
Andrew J. Swampillai · Ali. Khanan Kaabneh · Nabil E. Habib · Catriona Hamer • Phillip J. Buckhurst
}

Published online: 17 January 2020

(c) The Author(s), under exclusive licence to The Royal College of Ophthalmologists 2020

\section{Correction to: Eye}

https://doi.org/10.1038/s41433-019-0744-0
In the original publication, the second author name was spelt incorrectly. The author name Khanan Kaabneh has been corrected in the HTML and PDF of the original article. 